\title{
Análisis del agente lesivo en la baja de combate. Experiencia de la Sanidad Militar española desplegada en Herat (Afganistán).
}

\author{
Navarro Suay R. ${ }^{1}$, Hernández-Abadía de Barbará A. ${ }^{2}$, Gutiérrez Ortega C. ${ }^{3}$, Tamburri Bariain R. ${ }^{1}$, Bartolomé \\ Cela E. ${ }^{4}$, Gilsanz Rodríguez F. ${ }^{5}$ \\ Sanid. mil. 2011; 67 (1): 18-24; ISSN: 1887-8571
}

\begin{abstract}
RESUMEN:
Introducción: El conocimiento sobre aspectos relacionados con el armamento mejoran el diagnóstico y el tratamiento global del herido, sobretodo en el caso del médico militar. Se exponen los agentes lesivos de los principales conflictos recientes. El objetivo del estudio es el análisis del agente lesivo de las bajas atendidas en el Hospital Militar (ROLE 2) de Herat (Afganistán) entre 2005 y 2008. Material y método: Se ha diseñado un estudio descriptivo, transversal sobre todo el personal civil y militar que hubiese recibido herida por arma de fuego o por artefacto explosivo en la Región Oeste de Afganistán, y fuese atendido por el ROLE 2 español de Herat (Afganistán) durante los años 2005-2008. Resultados: El número total de casos analizados fue de 256. La mayor parte de los pacientes fueron heridos por artefacto explosivo, como los «Improvised Explosive Device» (IEDs) ( $n=183,71 \%$ ), mientras que el resto presentó lesiones por proyectiles de arma de fuego $(n=73,29 \%)$. Conclusiones: En nuestro estudio, el mecanismo lesivo predominante ha sido el explosivo, seguido de las armas de fuego. Este dato es semejante al obtenido en otros estudios anglosajones sobre las bajas en combate durante los conflictos en Irak y Afganistán.
\end{abstract}

PALABRAS CLAVE: Baja, Letalidad, Generación de bajas, Arma de fuego, Explosivo, Guerra, Sanidad militar.

\section{Analysis of the injuring agent in the combat casualty. The Spanish Medical Service experience in Herat (Afghanistan) SUMMARY:}

Introduction: The medical officer's knowledge about armament-related aspects improves the diagnosis and medical care of the casualty. In this article the injuring agents in the main recent conflicts are presented. The objective of this study is the analysis of the injuring agent in the casualties received in the military hospital in Herat (Afghanistan) between 2005 and 2008. Materials and methods: A descriptive, transversal study was carried out including all civilian and military personnel wounded by firearms or explosive devices in the West Region of Afghanistan, and taken care of in the Spanish Role 2 in Herat (Afghanistan) during the years 2005-2008. Results: The total number of cases was 256. The majority of the patients were wounded by explosive devices, as the «Improvised Explosive Devices» (IEDs) ( $n=183$; $71 \%)$, while the rest suffered gunshot wounds $(n=73 ; 29 \%)$. Conclusions: In our study the predominant mechanism of injury has been the explosive one, followed by firearms. These data are similar to those quoted in other English language reports about combat casualties in the Iraq and Afghanistan conflicts.

KEY WORDS: Low, Lethality, Casualty generation, Firearm, Explosive, War, Medical Service.

\section{INTRODUCCION}

Tradicionalmente el médico ha desconocido muchos de los aspectos relacionados con el armamento, quizás por creer que su conocimiento no tiene ningún efecto terapéutico sobre el enfermo o incluso porque éticamente es cuestionable; sin embargo, hay razones para contradecir esta actitud, como son que un médico pue-

${ }^{1}$ Cap. Médico. Escuela Militar de Sanidad. Madrid. España.

${ }^{2}$ Cte. Médico. Hospital Central de la Defensa Gómez Ulla. Unidad de Telemedicina. Madrid. España.

${ }^{3}$ Hospital Central de la Defensa Gómez Ulla. Servicio de Calidad y Medicina Preventiva. Madrid. España.

${ }^{4}$ Tcol. Médico. Hospital Central de la Defensa Gómez Ulla. Unidad de Cuidados Intensivos. Madrid. España.

${ }^{5}$ Universidad Autónoma de Madrid. Departamento de Cirugía. H.U. La Paz. Servicio de Anestesiología y Reanimación. Madrid. España.

Dirección para correspondencia: $\underline{\mathrm{r} \text { navarro suay@yahoo.es. }}$

Recibido: 16 de febrero de 2010

Aceptado: 29 de junio de 2010 de mejorar el tratamiento quirúrgico si comprende el daño tisular provocado por una bala, o que puede enfrentarse a la necesidad de extraer un proyectil no explosionado del interior del cuerpo de un paciente. Es recomendable conocer términos que valoren la efectividad del armamento y analizar los agentes lesionantes de los últimos conflictos.

En la actualidad, el Cuerpo Militar de Sanidad español se encuentra desplegado en el exterior en un buen número de Operaciones Internacionales. De todas ellas, la Operación ISAF (Internacional Security Assistance Force) en Afganistán es la que cuenta con una mayor dotación de personal, instalaciones y medios sanitarios. Concretamente, el Hospital Militar (ROLE 2) español ubicado en la ciudad de Herat (Afganistán) es el que está recibiendo pacientes que han sufrido heridas de arma de fuego o por explosivos (IED) y es considerado como la instalación sanitaria con más capacidad de toda la región Oeste de ese país ${ }^{1}$.

Este artículo tiene como objetivo el estudio del agente lesivo de las bajas atendidas en el Hospital Militar de Herat (Afganistán) durante el periodo comprendido entre 2005 y 2008. 
Tabla 1. Bajas distribuidas por sexo (n y \%) y por agente lesivo.

\begin{tabular}{|lcccccccc|}
\hline & $\mathbf{n}$ & $\mathbf{\%}$ & Explosivo (n) & Explosivo (\%) & Arma de fuego (n) & Arma de fuego (\%) & $\begin{array}{c}\text { Sexo por } \\
\text { Explosivo (\%) }\end{array}$ & $\begin{array}{c}\text { Sexo por arma } \\
\text { de fuego (\%) }\end{array}$ \\
\hline Hombre & 246 & 96 & 175 & 95,6 & 71 & 97,2 & 71 & 29 \\
Mujer & 10 & 4 & 8 & 4,4 & 2 & 2,8 & 80 & 20 \\
Total & 256 & 100 & 183 & 100 & 73 & 100 & - & - \\
\hline
\end{tabular}

\section{MATERIAL Y MÉTODOS}

Se ha diseñado un estudio descriptivo, transversal sobre todo el personal civil y militar que hubiese recibido herida por arma de fuego o por artefacto explosivo en la Región Oeste de Afganistán, y fuese atendido por el ROLE 2 español de Herat (Afganistán) durante los años 2005-2008.

Se empleó como criterio de inclusión todas aquellas personas que hubiesen sufrido una herida de arma de fuego o lesiones por artefacto explosivo, tanto sobre el terreno como durante un vuelo. No se emplearon criterios de exclusión.

Las variables incluidas en el estudio fueron independientes (sociodemográficas y de control: sexo, edad, civil/militar, zona geográfica de procedencia, transporte sanitario) y dependientes (agente lesivo-politómica: arma de fuego, explosivo, arma de fuego más explosivo-).

Para la realización del estudio se revisaron 12.256 historias clínicas, y la recopilación de los datos se llevó a cabo mediante una ficha de recogida de datos realizada para este trabajo.

El método estadístico empleado fue el descriptivo. Para variables categóricas se emplearon sus frecuencias absolutas y relativas en tantos por ciento $(\%)$.

\section{RESULTADOS}

Durante el periodo del estudio, se recopilaron un total de 256 bajas $(n=256)$ que habían sufrido lesiones diversas producidas durante enfrentamientos armados.

La mayor parte de los pacientes fue herido por artefacto explosivo, como los IEDs ( $n=183 ; 71 \%)$, mientras que el resto, presentó lesiones por proyectiles de arma de fuego $(n=73 ; 29 \%)$. Ver figura 1.

La mayoría de las bajas fueron varones $(n=246 ; 96 \%)$, únicamente $(n=10 ; 4 \%)$ eran mujeres (tabla 1$)$.

\section{Mecanismolesional (\%)}

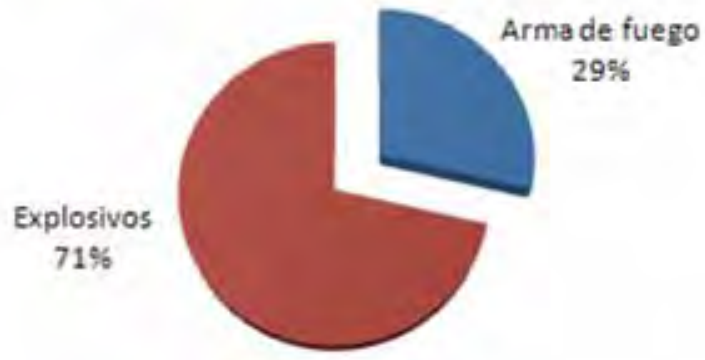

Figura 1. Bajas producidas por artefacto explosivo y por arma de fuego $(\%)$.
Casi la mitad de las bajas ( $\mathrm{n}=101 ; 39 \%)$ tenían entre 25 y 29 años, constituyendo este grupo el $39 \%$ de los lesionados por explosión, y el 39\% de los afectados por arma de fuego. Había más proporción de niños con heridas producidas por explosión que por arma de fuego (Figura 2).

El grupo con más bajas atendidas ha sido el «Afghan National Army» (ANA) ( $\mathrm{n}=112 ; 44 \%)$.

El medio de evacuación más empleado ha sido «aeroevacuación medicalizada» (MEDEVAC) ( $\mathrm{n}=194 ; 76 \%)$, constituyendo el método más frecuente, tanto en las bajas por explosivos $(n=134 ; 73 \%)$ como en las bajas por herida de arma de fuego $(n=60 ; 82 \%)$. No se observan, en la muestra estudiada diferencias en cuanto a la necesidad de evacuación a escalones superiores dependiendo del agente causal $(\mathrm{p}=0,216)$.

\section{DISCUSIÓN}

Intentamos aprovechar este estudio para incrementar el conocimiento en un tipo de paciente traumático que afortunadamente, no es frecuente en España, y de esta manera no sólo mejorar la calidad asistencial a futuras bajas, sino también extrapolar al mundo civil las lecciones aprendidas en el campo militar.

El médico militar, debe tener una instrucción completa en esta área, ya que como facultativo ha de conocer la fisiopatología de las lesiones por arma de fuego y por explosivos y como oficial tiene la obligación de defender a sus pacientes en las unidades sanitarias desplegadas en Zona de Operaciones. Analizando las heridas puede llegar a reconocer el empleo de nuevas armas por parte del enemigo, e incluso colaborar en el diseño de medidas de protección para los soldados.

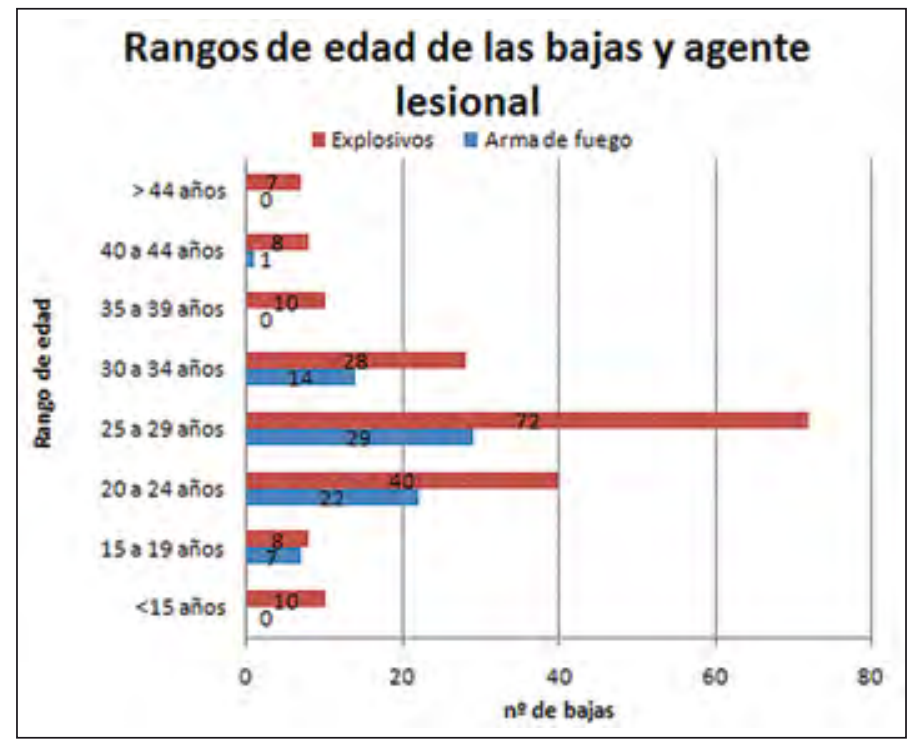

Figura 2. Rango de edad de las bajas y agente lesivo (n). 
Tabla 2. Distribución de heridos por arma a lo largo de las guerras del siglo $X X(\%)^{6,7}$.

\begin{tabular}{|lccc|}
\hline & Bala & Metralla & Varios \\
\hline I Guerra Mundial & 39 & 61 & - \\
II Guerra Mundial & 10 & 85 & 5 \\
Corea & 7 & 92 & 1 \\
Vietnam* & 52 & 44 & 4 \\
Borneo* & 90 & 9 & 1 \\
Irlanda del Norte* & 55 & 22 & 20 \\
Malvinas & 32 & 56 & 12 \\
Líbano & 20 & 60 & 20 \\
\hline
\end{tabular}

* Conflictos en donde predominan las bajas por bala.

Uno de los índices más comunes para valorar la efectividad del armamento, es el concepto de muerte provocada por éste (posibilidad de que una baja fallezca o sea herida por un arma). Desde un punto de vista sanitario, debemos definir dos conceptos relacionados con este término:

- Letalidad: Probabilidad de que una baja muera o tenga heridas graves provocadas por el empleo del arma en una ocasión. Históricamente, desconocemos en la mayoría de las ocasiones cuántos soldados fueron heridos por el uso de un arma, y de ellos cuántos murieron. Esto se debe principalmente a los siguientes hechos:

- Recogida de datos insuficiente. Incluso en el siglo XX, no se realizaba la autopsia a todos los fallecidos en combate para confirmar que la causa de muerte había sido provocada por un arma determinada.

- Varias causas de fallecimiento. Una misma baja podía estar afectada por varias armas a la vez. Ej.: proyectil de bala y metralla.

- No se diferencia entre los fallecidos antes de ser atendidos por personal sanitario y los que mueren tras aplicárseles algún cuidado médico.

- Es necesario comparar combates contemporáneos entre sí para poder establecer diferencias en cuanto a la letalidad. Ej.: durante la Guerra Civil norteamericana la tasa de mortalidad de un herido con fractura femoral conminuta por bala era de un $50 \%$ y el mismo tipo de herida en la Guerra de Vietnam provocaba una tasa de mortalidad de $1,4 \%{ }^{2}$. Sería del todo incorrecto suponer que la letalidad de un proyectil en la Guerra de Vietnam es inferior a los empleados durante la Guerra Civil norteamericana, y no contemplar los avances médicos realizados durante los aproximados 100 años que separan ambos conflictos.

- Generación de bajas: Número de personas que pueden verse afectadas por el empleo del arma en una ocasión. En lo primero que piensa el soldado cuando maneja un arma es en su efectividad (capacidad de un arma para herir o para matar). Sin embargo, hay que tener en cuenta las siguientes consideraciones:

- El número de bajas generadas por un arma, dependerá no sólo de sus características, sino también del número de bajas potenciales (población de riesgo). Ej.: Un pelotón de soldados con elementos de protección pasiva (casco o chalecos antifragmentos) que viajen en un vehículo blindado, sufrirán menos bajas que el personal civil sin ningún tipo de protección.

- Para generar bajas, el arma debe ser empleada con efectividad. Ej.: No es lo mismo que un proyectil de artillería caiga sobre la arena de una playa que sobre un edificio en el que viven muchas personas.

- A excepción de las bombas atómicas, no existe información fiable del número de veces que se emplea un arma para provocar bajas. Ej.: Se estima que en la II Guerra Mundial para provocar un herido en el bando contrario era necesario disparar 10.000 balas (1/10.000), sin embargo los francotiradores durante la Guerra de Vietnam emplearon 1,5 balas para conseguir el mismo objetivo $(1 / 1,5)^{3}$.

Si nosotros comparamos una granada de mano empleada en la II Guerra Mundial (letalidad 0,06 / generación de bajas 6-8), la bomba atómica de 12 kilotones arrojada en Hiroshima en 1945 (letalidad 0,5 / generación de bajas aproximadamente 144.000) y una espada utilizada en las Guerras Médicas (letalidad 0,95 / generación de bajas 1), obtenemos un claro ejemplo de la importancia de aclarar ambos términos ${ }^{3-5}$.

Se conoce que el cómputo de bajas por bala y por metralla difiere en cada conflicto. La Tabla 2 muestra como en general, los combates entre diferentes ejércitos (I Guerra Mundial y II Guerra Mundial) originan un mayor número de bajas por metralla, mientras que en el combate urbano, en la jungla o en enfrentamiento con terroristas, predominan las bajas por bala. Este hecho se confirma por medio de los datos recogidos en artículos de diversos autores ${ }^{8-19}$.

A pesar de que la mayoría de bajas en el combate moderno son provocadas por metralla, tenemos que tener en cuenta que este hecho es relativamente reciente, ya que en las guerras napoleónicas y durante los conflictos del S. XIX el principal agente lesional era el arma de fuego ${ }^{19,20}$.

A continuación se analiza el agente lesional en los principales conflictos del siglo XX y XXI.

\section{Guerra Mundial (1914-1918)}

Desde la I Guerra Mundial, la Artillería ocupó un papel predominante, provocando que aumentaran el número de heridas por metralla. (Tabla 3)

Esta estadística describe importantes diferencias entre bandos. Esto puede ser debido a:

- Recogida incompleta de los datos. Ej.: El 39\% de los fallecidos en el bando alemán a causa de heridas de bala, están basados en un análisis de 14.486 bajas, que suponen menos del 1\% de todas las bajas alemanas durante la I Guerra Mundial.

- Empleo de distintos tipos de táctica durante el combate. Ej.: Los alemanes pudieron emplear la Artillería tanto en operaciones de defensa como de ataque, mientras que las tropas norteamericanas se basaron en mayor medida en el empleo de armas propias de la Infantería.

Tabla 3. Bajas por nacionalidad y por agente lesivo ${ }^{21}$.

\begin{tabular}{|c|c|c|c|c|c|c|}
\hline & \multicolumn{2}{|c|}{ Alemanes 1914-1917 } & \multirow{2}{*}{$\frac{\text { Británicos }}{\text { Heridos }}$} & \multicolumn{2}{|c|}{ Norteamericanos 1918} & \multirow{2}{*}{$\frac{\text { Franceses } 1918}{\text { Heridos }}$} \\
\hline & Heridos & Muertos & & Heridos & Muertos & \\
\hline Balas & $51 \%$ & $39 \%$ & $39 \%$ & $14 \%$ & $8 \%$ & $30 \%$ \\
\hline Fragmentos* & $46 \%$ & $56 \%$ & $61 \%$ & $85 \%$ & $92 \%$ & $58 \%$ \\
\hline
\end{tabular}

* Metralla, granadas y bombas. Se desconocen los datos de fallecidos británicos y franceses en esta serie. 
II Guerra Mundial (1939-1945)

\section{Frente ruso-alemán. 1944}

Al estudiar las bajas alemanas en el frente ruso comprobamos que algunas armas como las minas anticarro provocaron una alta mortalidad (69\%) y por tanto una baja tasa de heridas leves $(9 \%)$. Sin embargo las granadas de mano ocasionaban pocas muertes (17\%), pero una alta tasa de heridos leves (65\%) que necesitaban apoyo médico ${ }^{22}$.

Las heridas por armas de Infantería, presentaban una letalidad del 0,3 , mientras que la metralla procedente de la artillería y de los morteros provocaba una letalidad entre el 0,08-0,1922.

\section{Frente del Pacífico. Campaña de Bougainville. Febrero-Abril 1944}

Si analizamos las bajas de este frente, comprobamos el alto grado de letalidad obtenido por las heridas producidas por ametralladora $(0,58)$. La posible explicación se debe a que esta arma suele provocar múltiples heridas en un mismo soldado. La letalidad de las heridas por proyectiles disparados por fusil fue de 0,32 , muy superior a la letalidad de los proyectiles de mortero $(0,12)$ y a la de la artillería $(0,11)^{23}$. La explicación puede razonarse por lo complicado del despliegue de baterías de Artillería en un ambiente tropical como el del Pacífico. En esta situación, las balas producen más muertes. Sin embargo la metralla de mortero y artillería provoca la mayoría del esfuerzo quirúrgico, al igual que lo que ocurrió en el frente europeo y del Norte de África (a pesar de que en estos últimos entornos, predominaron operaciones mecanizadas pesadas).

\section{Experiencia británica en el Desembarco de Normandía. Junio-Julio 1944}

Durante las 6 semanas que duró la Campaña de Normandía, los británicos sufrieron 3.609 del total de 50.000 bajas sufridas durante la II Guerra Mundial ${ }^{24}$. Los despliegues tácticos fueron heterogéneos: anfibio, urbano y con fuerte implicación de vehículos blindados (Operación Goodwood). Alrededor de 2/3 de las bajas hospitalizadas fueron por metralla, mientras aproximadamente el 1/3 restante fueron por heridas de arma de fuego. Curiosamente, la distribución de bajas por tipo de proyectil (no por arma) fue similar a las bajas hospitalizadas en la Campaña de Bougainville.

La serie muestra claramente como la letalidad por proyectiles de arma de fuego (39\%) es superior a la producida por cualquier otra arma.

Este estudio no analiza el impacto de la asistencia médica en el frente. Sin embargo se conoce que el $83 \%$ de las muertes ocurrieron antes de recibir apoyo médico ${ }^{24}$.

\section{Bajas norteamericanas durante la II Guerra Mundial}

La serie de Reister ${ }^{25}$ muestra que las letalidades de las heridas por fusil $(0,38 \%)$ y por metralla $(0,26 \%)$, son similares a las obtenidas en el estudio británico sobre las bajas en el Desembarco de Normandía.

Aproximadamente 1/3 de los fallecidos en combate y 1/5 de los hospitalizados fueron a causa de las heridas por proyectil de fusil. $\mathrm{Si}$ analizamos todos los Teatros, descubrimos que lo más significativo es el elevado número de bajas por balas en Suroeste del Pacífico ( $52 \%$ de fallecidos y $32 \%$ de los hospitalizados $)^{25}$. No hay duda de que esto es consecuencia de las peculiaridades tácticas acontecidas en este ambiente. Por otro lado, el predominio de combates entre Unidades Mecanizadas en Europa, provocó una disminución de la importancia de la Infantería y de las heridas provocadas por balas en este Teatro (balas 33\%, metralla 52\%) ${ }^{25}$.

Sería interesante contemplar el número de bajas sufridas en el Ejército alemán en Francia y Alemania, para compararlas a las de las fuerzas aliadas.

\section{Guerra de Corea (1950-1953)}

\section{Bajas norteamericanas}

La letalidad de las heridas por bala $(0,26 \%)$ es $1 / 3$ menor a la obtenida en conflictos anteriores, sin embargo la provocada por la artillería $(0,22 \%)$ sí es similar ${ }^{26}$.

En esta guerra se recoge que la mitad de los heridos durante operaciones de retirada y repliegue fueron por arma de fuego (37\% durante Julio-Noviembre de 1950). Sin embargo, cuando las fuerzas se encontraban atrincheradas en operaciones defensivas, las bajas por arma de fuego disminuyeron a un 15\% (11\% durante Octubre 1951-Julio 1953). Estos datos son similares a los encontrados durante la I Guerra Mundial, también conocida como la «Guerra de las Trincheras» ${ }^{26}$.

\section{Guerra de Vietnam. (1965-1970)}

\section{Bajas norteamericanas}

Las series recogidas en la Tabla 4 muestran unos resultados similares a los obtenidos en la II Guerra Mundial en la Campaña de Bougainville.

El entorno tropical es parecido y el tipo de combate es semejante al desarrollado con anterioridad en el frente del Pacífico de la II Guerra Mundial (baja intensidad con operaciones llevadas a cabo por Infantería Ligera). Eran frecuentes las acciones llevadas a cabo por pequeñas unidades, en donde los tiroteos eran la forma más frecuente de combate. Las tropas norvietnamitas y el Viet Cong no empleaban baterías de artillería convencional, pero sí morteros y granadas, produciendo baja letalidad pero un gran número de bajas (letalidad de bala $43 \%$, letalidad de metralla $15 \%)^{28}$.

Tabla 4. Bajas norteamericanas en la Campaña de Bougainville y Vietnam $^{23,27}$

\begin{tabular}{|lcc|}
\hline \multicolumn{1}{c}{ Arma } & Bougainville (\%) & Vietnam (\%) \\
\hline Bala & 33,3 & 30 \\
Mortero & 38,8 & 19 \\
Artillería & 10,9 & 3 \\
Granada & 12,5 & 11 \\
Mina terrestre/trampa explosiva & 1,9 & 17 \\
RPG* & - & 12 \\
Varios & 2,6 & - \\
\hline
\end{tabular}

* RPG: Rocket propelled grenada 
La mayor parte de soldados y marines fallecidos fueron a consecuencia de heridas por bala durante enfrentamientos directos. Sin embargo, la mayoría de heridos fueron por ataques con RPG (Rocket Propelled Grenade - granada propulsada-) sobre los campamentos ${ }^{27}$. Por tanto la situación táctica fue fundamental para comprender el tipo de lesión provocada. Mientras que en la ofensiva eran más frecuentes las bajas por bala, durante la fase de defensa en las bases el principal agente lesivo fue la metralla, ya que era el único método empleado por las fuerzas locales para producir daño a los norteamericanos.

Se desconoce la distribución de bajas y tipo de lesiones sufridas en el Ejército norvietnamita y en el Viet Cong. El porcentaje de supervivencia tras sufrir heridas por bala probablemente fuese escaso. La gran mayoría de sus bajas seguramente fuesen originadas por metralla, procedente de artillería y de ataques aéreos.

\section{Irlanda del Norte. (1969-Presente)}

\section{Bajas británicas}

La información recogida en la serie de Owen-Smith ${ }^{29}$ probablemente sea la representativa de los atentados terroristas en ambiente urbano. Muestra claramente que las armas de fuego provocan el $54 \%$ de los fallecidos. La segunda causa de muerte fue el blast, procedente de los artefactos explosivos.

Además, las balas causan una proporción mayor de heridos que en el campo de batalla. Este estudio es particularmente valioso ya que permite comparar la letalidad en ambiente militar $(0,37$, proyectiles de alta velocidad) y en ambiente civil $(0,075$, proyectiles de baja velocidad).

\section{Guerra Libano-israelí (1982)}

\section{Bajas israelíes}

Los datos según el estudio de $\operatorname{Rogov}^{30}$ muestran que únicamente fallecieron el $11 \%$ de las bajas por metralla, mientras que en la serie publicada por Besser ${ }^{31}$ esta cifra asciende a $21 \%$. A pesar de analizar el mismo conflicto, la diferencia se puede deber a la forma de recogida de datos. De cualquier modo, vuelve a observarse el hecho de que la letalidad de las heridas por bala es mayor que la de metralla ( $31 \%$ según $\operatorname{Rogov}^{30}$ y 28\% según Besser ${ }^{31}$ ).

Del mismo modo, si analizamos las bajas dependiendo de lugar donde se han producido (ambiente urbano y no urbano) encontramos que en el primero, hay una alta incidencia de bajas por artillería y morteros (33\%), mientras que en el segundo, destaca el número de bajas producidas por fusil y pistolas $(21 \%)^{31}$. Este patrón difiere del combate urbano acontecido en la ciudad de Stalingrado en la II Guerra Mundial, en donde las bajas por granada y por bombardeo aéreo originaron la mayor parte de las víctimas ${ }^{32}$.

\section{Guerra de las Malvinas (1982)}

Las bajas británicas en este conflicto fueron provocadas por los siguientes agentes lesivos $(n=233$, metralla $45 \%$, bala $31,8 \%$, mina $10,8 \%$ y varios $12,4 \%)^{33}$.

\section{Guerra en Bosnia-Herzegovina (1992-95)}

Las bajas en este conflicto atendidas por la Sanidad Militar española quedan reflejadas en los estudios de Villanueva $(n=39 ; 92 \%$ por explosivos $)^{34}$ y Villalonga $(n=42 ; 46 \% \text { por explosivos })^{35}$.

\section{Guerra en Irak y Afganistán (2001-actualidad)}

La experiencia obtenida sobre los mecanismos lesivos en las bajas atendidas durante los conflictos de Irak y Afganistán se muestran en la Tabla 5.

Hemos analizado artículos publicados en revistas científicas sobre bajas procedentes de Irak y de Afganistán que contengan el parámetro anteriormente estudiado del mecanismo lesivo. A pesar

Tabla 5. Distribución por autores del mecanismo lesivo empleado ${ }^{36-50}$

\begin{tabular}{|lcccc|}
\hline Autor & Año & Guerra & N & Mecanismo lesivo \\
\hline Peoples $^{36}$ & $2001-2002$ & Afganistán & 224 & Explosivos (49\%) \\
Fox $^{37}$ & $2001-2004$ & Irak y Afganistán & 1524 & Explosivos $(64 \%)$ \\
Owens $^{38}$ & $2001-2005$ & Irak y Afganistán & 1281 & Explosivos (75\%) \\
Beekley $^{39}$ & $2002-2003$ & Afganistán & 90 & Arma de fuego (34\%) \\
Beitler $^{40}$ & $2002-2003$ & Afganistán & 204 & Explosivos (36\%) \\
Hinsley $^{41}$ & 2003 & Irak & 482 & Explosivos (62\%) \\
Zouris $^{42}$ & 2003 & Irak & 279 & Explosivos (75\%) \\
Montgomery43 $^{43}$ & Irak & 294 & Arma de fuego (39\%) \\
Patel $^{44}$ & 2003 & Irak & 154 & Arma de fuego (+ frecuente) \\
Reavley $^{45}$ & 2003 & Irak & 66 & Explosivos (68\%) \\
Colombo $^{46}$ & $2003-2004$ & Irak y Afganistán & Explosivos (58\%) \\
Gondusky $^{47}$ & $2003-2007$ & Irak & 125 & Explosivos (90\%) \\
Woodward $^{48}$ & 2004 & Irak & 9289 & Explosivos (54\%) \\
Murray $^{49}$ & $2004-2007$ & Irak & 355 & Explosivos (78\%) \\
Owens $^{50}$ & $2003-2004$ & Irak y Afganistán & 1566 & Explosivos (78\%) \\
\hline
\end{tabular}




\section{Análisis del agente lesivo en la baja de combate. Experiencia de la Sanidad Militar española...}

de ser conflictos diferentes, presentan gran similitud desde un punto de vista logístico-operativo y asistencial.

En nuestra serie, el mecanismo lesivo predominante ha sido el explosivo, seguido de las armas de fuego. Este dato es semejante al obtenido en prácticamente la totalidad de estudios anglosajones que analizan las bajas atendidas en los conflictos más recientes $^{36-38,40-42,45-50}$. Este hecho también es característico del enfrentamiento entre tropas profesionales e insurgentes, ocurrido en ambiente urbano, desértico o montañoso y en la denominada «guerra asimétrica» ${ }^{36-50}$.

Entre otros factores, se puede destacar que las medidas de protección pasiva (blindaje de vehículos, casco y chaleco antifragmentos) y la rápida evacuación hasta una instalación médica, reducen la morbimortalidad en los heridos que han sufrido lesiones por arma de fuego y por explosivos (en inglés Improvised Explosive Device, IED).

\section{CONCLUSIONES}

En nuestro estudio, el mecanismo lesivo predominante ha sido el explosivo, seguido de las armas de fuego.

Nuestros resultados sobre los agentes lesionales son semejantes a los obtenidos en la mayoría de estudios anglosajones que estudian las bajas en combate de los conflictos de Irak y Afganistán.

\section{BIBLIOGRAFÍA}

1. Navarro R, Bartolomé E, Jara I, Oreja A, González G. Capacidades y asistencia sanitaria realizada por el ROLE-2 español en la FSB de Herat (Afganistán) desde febrero a julio del 2007. Sanid. Mil. 2008; 64 (2): 98-104.

2. Oughterson A, Hull H, Sutherland F, Greiner D. Study on wound ballistics. Bougainville campaing. En J.C Beyer.Wound ballistics, $1^{\text {a }}$ ed. Washington, DC. Office of the Surgeon General, Department of the Army; 1962. p. 281-463.

3. Dupuy TN. The evolution of weapons and warfare. Indianapolis: The BobbsMerrill Company Inc; 1982.

4. Adamson, PB. A comparison of ancient and modern weapons in the effectiveness of producing battle casualties. J. Roy Army Med Cps 1982. 123: 93-103.

5. Cutting P, Surgery in the Camp. En Mahoney P, Ryan J, Brooks A, Schwab C, editors. Ballistic Trauma. $2^{\text {a }}$ ed. Springer; 2004. 585-91.

6. Cooper G, Gotts P. Ballistic Protection. En Mahoney P, Ryan J, Brooks A, Schwab C, editors. Ballistic Trauma. $2^{\mathrm{a}}$ ed. Springer; 2004. 67-89.

7. Salas JH. Tratamiento de las heridas craneoencefálicas en la guerra. Rev Cubana Med Milit 1998; 27(2): 113-19.

8. Johnson DE, Panijayanon P, Lumjiak S, Crum JW, Boonkrapu P. Epidemiology of combat casualties in Thailand. J Trauma 1981; 21(6): 486-8.

9. Leedham CS, Blood Ch G. A descriptive analysis of wounds among U.S marines treated at second echelon facilities in the Kuwait theater of operations. Milit Med 1993; 158: 508-12.

10. Danon YL, Nili E, Dolev E. Primary treatment of battle casualties in the Lebanon war 1982. Israel J of Med Sci 1984; 20: 300-2.

11. Rautio J, Paavolainen P. Afghan war wounded: experience with 200 cases. J Trauma 1988; $28: 523-5$.

12. Al-Harby SW. The evolving pattern of war related injuries from the Afganistan conflict. Milit Med 1996; 161-4.

13. Bhatnagar MK. Trauma in the Afghan guerrilla war: effects of lack access to careSurgery 1989; 105: 699.

14. Prgomet D. Puntaric D. Organization and work of medical service during 1992 military operations in north Bosnia (Bosanska Posavina). Milit Med 1996; 161: 661-4.

15. Atias Nikolov V. Organization and work of the war hospital in Sturba near the town of Livno, Bosnia and Herzrgovina. Milit Med 1995;160: 62-9.
16. Manejo integral del herido de guerra de la lucha irregular en el teatro de operaciones militares de Nicaragua en el bienio 1984-1986. En Primera Conferencia Científica de los Servicios Médicos. Ejército Popular Sandinista; Nicaragua: Apanas Jinotega;1986. Menéndez López JR.

17. Nassoura Z. Trauma management in a war zone: the Lebanese war experience. J Trauma 1991; 31: 1596-9.

18. Brandvold B, Levi L, Feinsod M, George ED. Penetrating craneocerebral injuries in the Israeli involvement in the Lebanese conflict, 1982-1985. J Neurosurg 1990; 72: 15-21.

19. A. Ballesteros Fernández. La Sanidad Militar durante la Guerra de la Independencia. Sanid. Mil. 2008; 64(4): 235-44.

20. Clifton F.From civil war to world war,1865-1917. En: Greenwood JT. Medics at war: military medicine from colonial times to the $21^{\text {st }}$ century. Maryland: Association of the United States Army; 2005. P. 45-62.

21. Barnett G. Caring for the Casualties.WWII History 2008; 4: 40-7.

22. Mueller-Hillebrand, B. Statistisque system. Washington,DC.United States Army Historical Division Study No. PC 011.

23. Oughterson A, Hull H, Sutherland F, Greiner D. Study on wound ballistics. Bougainville campaing. En J.C Beyer.Wound ballistics, $1^{\mathrm{a}}$ ed. Washington, DC. Office of the Surgeon General, Department of the Army; 1962. p. 281-463.

24. Crew F. Campaigns: North-west Europe. En: The Army Medical Service. Londres: Her Majesty's Stationery Office; 1962.Vol 4. p. 597-608.

25. Reister F. Medical statistics in World War II. Washington,D.C: Office of The Surgeon General, Department of Army; 1975.

26. Burris D, et al. Weapons effects and parachute injuries. En Szul A. Emergency War Surgery. $3^{\mathrm{a}}$ ed. Borden Institute Walter Reed Medical Center; 2004. 1.1-1.11.

27. Wound Data and Munitions Effectiveness Team. Evaluation of wound data and munition effectiveness in Vietnam (Final Report). Alexandria,VA: Defense Documentation Center of the Defense Logistics Agency. 1970; In Vol. 3, table 4, p. C-7.

28. Bellamy, R. The Casualty. Bethesda, Maryland: Uniformed Services University of the Health Science University; 1987.

29. Owen-Smith,M. A computerized data retrieval system for the wound of war. The Northern Ireland casualties. J. Roy. Army Med. Cps. 1980; 127: 31-54.

30. Rogov,M. Pathological evaluation of trauma in fatal casualties of Lebanon War. Israeli J. Med. Scien. 1982 20: 367-72.

31. Besser,Y. Military operation in urbanized terrain (MOUT) Thesis. Maryland: Uniformed Services University of the Health Science; 1985.

32. Bellamy R, Zajtchuk R. Assessing the effectiveness of conventional weapons. Conventional Warfare, ballistic, blast and burn injuries.1 ed. Textbook of Military Medicine Publications; 1991. 53-82.

33. Jackson DS, Batty CG, Ryan JM, McGregor. Army Field Surgical Experience. The Falklands War. JR Army Med Corps 153(S1): 44-7.

34. Villanueva Serrano S, Martínez Pérez JM, Herrera Morillas F, Hernández-Abadía de Barbará A. Bajas por munición explosiva. Experiencia española en la antigua Yugoslavia. Med Mil.1997; 53(4): 339-43.

35. Villalonga Martínez L.M. Sanidad Militar en Bosnia-Herzegovina. Med Mil 1996; 52(2): 177-80

36. Peoples G, Gerlinger T, Craig R, Burlingame B. Combat casualties in Afghanistan cared for by a single Foward Surgical Team during the initial phases of Operation Enduring Freedom. Mil Med. 2005; 170(6): 462-8.

37. Fox CJ, Gillespie DL, O'Donnell SD, Rasmussen TE, Goff JM, Jonson CA, etal. Contemporary management of wartime vascular trauma. J Vasc Surg, 2005; 41(4): 638-44.

38. Owens BD, Kragh JF, Macaitis J, Svoboda SJ, Wenke JC. Characterization of extremity wounds in Operation Iraqi Freedom and Operation Enduring Freedom. J Orthop Trauma. 2007; 21(4): 254-7.

39. Beekley A, Watts DM. Combat trauma experience with the United States Army 102nd Foward Surgical Team in Afghanistan. Am J Surg. 2004; 187(5): 652-4.

40. Beitler A, Wortmann G, Hofmann L, Goff J. Operation Enduring Freedom: the 48th Combat Support Hospital in Afghanistan. Mil Med. 2006; 171(3): 189-93.

41. Hinsley DE, Rosell PA, Rowlands TK, Clasper JC. Penetrating missile injuries during asymmetric warfare in the 2003 Gulf conflict. Br J Surg. 2005; 92(5): 637-42.

42. Zouris JM, Walter GJ, Dye J, Galarneau M. Wounding patterns for U.S. Marines and sailors during Operation Iraqi Freedom, major combat phase. Mil Med. 2006; 171(3): 246-52.

43. Montgomery SP, Swiecki CW, Shriver CD. The evaluation of casualties from Operation Iraqi Freedom on return to the continental United States from March to June 2003. J Am Coll Surg. 2005; 201(1): 7-12; discussion 12-3.

44. Patel TH, Wenner KA,Price SA, Weber MA, Leveridge A, McAtee SJ. US. Army Forward Surgical Team's experience in Operation Iraqi Freedom. J Trauma. 2004; 57(2): 201-7. 


\section{R. Navarro Suay, et al.}

45. Reavley P, Black J. Attendances at a Field Hospital emergency department during operations in Iraq November 2003 to March 2004 (Operation Telic III). J R Army Med Corp 2006; 152: 231-5.

46. Colombo CJ, Mount CA, Popa CA. Critical care medicine at Walter Reed Army Medical Center in support of the global war on terrorism. Crit Care Med. 2008 36(7 Supple): S388-94.

47. Gondusky J, Reiter M. Protecting Military Convoys in Iraq: An examination of Batlle Injuries Sustained by a Mechanized Battalion during Operation Iraqi Freedom II. Mil Med. 2005; 170(6): 546-9.
48. Woodward EB, Clouse WD, Eliason JL, Peck MA, Bowser AN, Cox MW, etal Penetrating femoropopliteal injury during modern warfare: experience of Balad Vascular Registry. J Vasc Surg. 2008; 47 (6): 1259-64.

49. Murray C, Reynolds J, Schroeder J, Harrison M, etal. Spectrum of care provided at an Echelon II Medical Unit during Operation Iraqi Freedom.Mil Med. 2005; 170(6): 516-520

50. Owens BD, Kragh JF, Wenke JC, Macaitis J, Wade CE, Holcomb JB. Combat wounds in operation Iraqi Freedom and operation enduring freedom. J Trauma. 2008; 64(2): 295-9. 